\title{
El aprendizaje colaborativo en la clase de Inglés: El caso del curso de Comunicación Oral I de la Sede del Pacífico de la Universidad de Costa Rica
}

\section{Collaborative Learning in the English Class: the Case of the Oral Communication I Course at Pacific Campus of the Universidad de Costa Rica}

\author{
Jonnathan Salas-Alvarado ${ }^{1}$ \\ Universidad de Costa Rica, Sede del Pacífico \\ Esparza, Costa Rica \\ josa325@hotmail.com \\ ORCID: orcid.org/0000-0001-6980-7089
}

Recibido: 7 octubre 2014 Aprobado: 22 octubre 2015 Corregido: 11 marzo 2016

\begin{abstract}
Resumen: El siguiente artículo consiste en una sistematización del proceso que se sigue para la aplicación de una estrategia didáctica diferente, producto de la experiencia vivida tras el curso de docencia universitaria de la Universidad de Costa Rica por parte de un profesor del curso de Comunicación Oral I, impartido en la sede del Pacífico de la Universidad de Costa Rica. Se describe la experiencia vivida al hacer uso de actividades relacionadas con el aprendizaje colaborativo. El artículo, además, describe a la población con la que se trabajó, las técnicas que se aplicaron, las razones por las cuales se utilizaron dichas técnicas y los resultados que dieron estas vistos desde la óptica del docente y del estudiantado. Se provee, también, un análisis acerca de las bondades, las limitaciones que tuvo la estrategia y su utilidad para el proceso de enseñanza-aprendizaje de una lengua. El aprendizaje colaborativo, basado en los resultados de esta estrategia tiene muchas ventajas que aportar a la educación.
\end{abstract}

Palabras clave: Aprendizaje colaborativo, estrategia de aprendizaje, Sede del pacífico, aprendizaje del inglés.

\begin{abstract}
This is an article that consists on the systematized process that is followed for the implementation of a different didactic strategy as a product of the experience lived in the "didáctica Universitaria" course of the University of Costa Rica by the instructor of Oral Communication I course that was been taught at the Pacific Campus of the University of Costa Rica. The experienced lived after using activities related to the collaborative learning technique is described. This article also describes the population that was used, the techniques applied, the reasons why those techniques were used and their result from the teacher's and the students' perspective. An analysis based on the strategy's advantages and limitations is provided as well as its utility in the language teaching-learning process. The collaborative learning, based on the results of this strategy, has many advantages to provide to education.
\end{abstract}

Key words: Collaborative learning, learning strategy, Pacific campus, English learning.

1 Máster en Ciencias de la Educación con Énfasis en la Enseñanza del Inglés. Profesor de inglés en la Sede del Pacífico de la Universidad de Costa Rica y en el Colegio Laboratorio del CUP. Coordinador del Proyecto de trabajo comunal TC-578: Mejoramiento de la calidad de vida de la población puntarenense y coordinador del proyecto ED-3091: Fortalecimiento de la enseñanza y aprendizaje del inglés. 


\section{Introducción}

De acuerdo con Quezada (2011), el inglés es una de las lenguas más habladas en el mundo y esto, según ella, se debe, por ejemplo, al poder hegemónico que han ejercido Estados Unidos y Gran Bretaña durante los dos últimos siglos. El origen exacto de las lenguas es un tanto difícil de establecer, debido a su constante evolución. El inglés, por su parte, pertenece a la gran familia de lenguas indo-europeas y, a su vez, de manera más específica pertenece, junto con el danés, el alemán y el sueco, a la familia de lenguas germánicas. Lo anterior no quiere decir que esta lengua se haya mantenido estática y aislada del resto del mundo. Muy por el contrario, este es un idioma que debido a diversas situaciones políticas, geográficas, históricas y culturales se ha visto obligado a tener contacto, algunas veces hasta forzoso, con otras lenguas que, eventualmente, la han ido moldeando hasta llegar a ser lo que es hoy.

Ahora bien, ¿cómo y cuándo nace el interés de los seres humanos occidentales en el aprendizaje de lenguas extranjeras?, ¿cuál es la razón de este fenómeno? Según Silva (2006), es durante la época clásica cuando Sócrates, Platón y Aristóteles inician discusiones en torno al origen de las lenguas; esto, desde un punto de vista muy filosófico. No es sino hasta el siglo III A.C cuando la Escuela de Gramática de Estoica considera el estudio de la lengua como una disciplina independiente de la filosofía.

A lo largo de la historia, la enseñanza-aprendizaje de las lenguas ha ido cambiando y evolucionando, muchas han sido las corrientes de pensamiento que han sido utilizadas en este proceso. Destacan, por ejemplo, la gramática tradicional, el conductismo que trabajaba con los preceptos de que la lengua era adquirida basándose en la imitación que hacen los niños y niñas e iba a ser promovida o censurada mediante refuerzos negativos o positivos (Silva (2006). Por otra parte, también se menciona al estructuralismo que se enfoca más en la forma de la lengua que en su uso.

Al volverse el inglés una lengua de suma importancia a nivel mundial en diversos campos de acción, es lógico que se inicie el interés de la población en general por aprenderlo y es por esta razón que empiezan a surgir diversos métodos de enseñanza que, efectivamente, también servían para enseñar otras lenguas. La importancia del inglés va creciendo poco a poco debido a la influencia de potencias económicas a nivel mundial, el caso de Inglaterra por un lado y Estados Unidos por otro, ambas naciones angloparlantes. Marín (2012) señala que, por ejemplo, a finales del siglo XIX "los ingleses controlaban el 50\% o más de las cosechas de café que exportaba Costa Rica” (Quesada, 1993, citado por Marín, 2012, p. 4). De la misma forma, "en los últimos años del siglo XIX y principios del XX, nos encontramos con la presencia de algunas compañías inglesas y norteamericanas” (Marín, 2012, p. 5). Costa Rica, en efecto, era solo una más de las naciones que sentía la necesidad de aprender inglés.

Inglaterra, por ejemplo, se destacó por su expansionismo acelerado y poderoso a través del mundo, de hecho, tal y como lo señala Jiménez (2010), el imperio británico "es el primero de la contemporaneidad, alcanzando una extensión de casi 22,5 millones de kilómetros cuadrados, que suponían más de la mitad de los territorios colonizados en aquellos momentos" (p. 4). Inglaterra, continúa Jiménez (2010), contaba con colonias en todos los continentes. Por tanto, el aprender inglés se volvía una necesidad para muchas personas alrededor del mundo y por ello se buscan métodos y estrategias para su enseñanza y aprendizaje. 
Un concepto de suma importancia en esta instancia es el de método, el cual se define como "un cuerpo de teoría verificado científicamente o una serie de procedimientos, medios generales o técnicas usadas de forma sistemática para lograr un objetivo" (Hernández, 2000, p. 141). Básicamente, se refiere a la manera en la que el personal docente acerca el conocimiento a sus estudiantes, este es "el camino para llegar a un resultado determinado" (Martín 2009, p. 61).

En relación con lo anterior, se puede decir que han surgido varios métodos a través de la historia, unos han probado ser efectivos y otros no, unos han sido criticados y han caído en desuso, otros han sido modificados y aún siguen siendo utilizados. Y es que según Martín (2009), visto desde un plano histórico-pedagógico, la evolución de métodos, técnicas, procesos en lo referente a la adquisición de una segunda lengua han tenido un papel preponderante en materia educativa. Martín (2009) considera que con la aparición del humanismo y el auge de nuevas lenguas modernas en una Europa renacentista, el estudio de estas lenguas así como "la perfección metodológica" (Martín 2009, p. 55) se enfrentó a un momento de eclosión; pero no sería sino hasta mucho tiempo después (siglo XX) cuando la revolución metodológica tendría inicio y se desarrollaría.

La enseñanza de las lenguas pasa por varios momentos a través de la historia de la humanidad, desde un humanismo que ve la educación como parte importante de la revolución del pensamiento renacentista; un humanismo en el cual el estudio del latín, griego y hebreo era esencial para acercarse al conocimiento atesorado desde el pasado.

Durante el siglo XVII en Occidente, el realismo pedagógico inicia su desarrollo. Según Martín (2009), este buscaba darle un uso a las cosas que se aprendían. En lo que respecta al siglo XVIII, el llamado "Siglo de las Luces", la evolución de la enseñanza de lenguas extranjeras tiene un auge, ya que es en este momento cuando esta encuentra cabida dentro del currículo académico; sin embargo, todo aún giraba en torno al latín y se trabajaba con los métodos que se utilizaban para enseñarlo. En este momento comienza la aparición y desarrollo de métodos.

De acuerdo con Hernández (2000, p. 142), en lo concerniente a la enseñanza de lenguas, es importante distinguir entre "métodos generales y específicos, tradicionales y contemporáneos, más sin hacer válidas estas distinciones".

Las metodologías de enseñanza de lenguas extranjeras han evolucionado con el tiempo; básicamente desde las perspectivas y teorías lingüísticas y psicológicas, todo progreso en esas áreas se refleja en las prácticas pedagógicas y en lo que respecta a los currículos en la enseñanza de idiomas (Muñóz, 2010).

Entre los métodos para la enseñanza de una lengua extrajera más comunes se destaca el método de gramática-traducción. Este se ha utilizado históricamente para la enseñanza de las lenguas clásicas; se basa en reglas gramaticales, la memorización de vocabulario, la traducción de textos, y otros tantos ejercicios escritos (Brown, 2001). La idea es, entonces, lograr la comprensión de textos mediante la traducción directa. Según Brown (2001) este método se aplica usando la lengua materna del estudiantado, el vocabulario se enseña de manera aislada y se valoran mucho las explicaciones gramaticales, ya que es esta la que provee las reglas necesarias para la comprensión de los textos. Este método, al estar 
enfocado más hacia las reglas gramaticales y la práctica de estas, no presenta características colaborativas. Quienes aprenden una lengua meta mediante este método se enfocan en sus prácticas y en la comprensión de reglas gramaticales y palabras.

Otro método es el directo. Tal y como es descrito por Brown (2001), busca simular la manera natural en la que las personas aprenden su lengua materna. Contiene mucha interacción social, un uso espontaneo de la lengua meta, no contempla traducción de una lengua a otra y no hay un análisis de las reglas gramaticales. Brown (2001) establece que este método se desarrolla principalmente en clases muy pequeñas, en donde existe atención individualizada e intensiva. Esta interacción no necesariamente implica colaboración entre quienes estudian la lengua, se busca más una relación más cercana entre el estudiantado y quien instruye sobre la lengua.

En el caso del método audio-lingual, conocido también como el método del ejército, cuyo origen, según lo describe Alcalde (2011), "está vinculado a la Segunda Guerra Mundial, ya que el ejército Americano necesitaba disponer de hablantes de varias lenguas extranjeras en poco espacio de tiempo" (p. 14). Este método, continúa Alcalde (2011), se caracteriza por ser "rápido y seguro para la adquisición de las lenguas" (p. 14). Se prioriza la expresión oral y la audición y además busca "la corrección lingüística y se trata de que el individuo aprenda el nuevo vocabulario por asociación de la palabra hablada y la imagen visual” (Hernández, 2000, p. 144).

La idea básica de este método, de acuerdo con Hernández (2000), es la repetición y da énfasis a ejercicios mecánicos y de repetición de patrones nativos. Brown (2001) establece que en este método hay una dependencia por la imitación y la repetición, la gramática se enseña de manera inductiva, el vocabulario enseñando depende del contexto en que se trabaje y da mucha importancia a la pronunciación. Este método (Brown, 2001) hace uso de cintas para grabar, laboratorios de idiomas y ayudas visuales; los estudiantes y las estudiantes trabajan para sí, por tanto el nivel de colaboración dentro de este método también es limitado o nulo.

La sugestopedia es "un intento, por medio de diversas vías (audios, traducciones, mímica, etc.) de activar las dos mitades del cerebro para el proceso de aprendizaje partiendo de la psicoterapia comunicativa y otras disciplinas psicoterapéuticas" (Alcalde, 2011, p. 17). Hernández (2000, citando a Pekelis, 1987) establece que la sugestopedia "enfatiza el desarrollo de una memoria exigua y expone al aprendiz a un conjunto de efectos psíquicos con el objetivo de sumergirlo en el contexto del idioma nuevo y hacerlo que se sienta con más libertad de hablar" (p. 146). Por tanto, tenemos, entonces, un método que busca promover el aprendizaje de una lengua extranjera en un ambiente relajado y sin presiones. Sin embargo, este método tampoco establece un ambiente colaborativo como una de sus premisas.

La vía silente o el método silencioso es descrito por Brown (2001) como un enfoque en el que el aprendizaje se facilita en tanto quien aprende descubra o crea en lugar de recordar y repetir lo que aprende, a su vez se promueve la resolución de problemas en relación con lo aprendido, se espera que quien aprende desarrolle independencia, autonomía y responsabilidad (Brown, 2001). Por su parte, Tejeda et al. (2005 citados por Álvarez, 2013) aseguran que este método tiene un "procedimiento uniforme en el aula; primero los sonidos, después las palabras y finalmente las oraciones que se enseñan a través de barras coloreadas de diferentes formas" (p. 17). Por tanto, este método busca que cada estudiante inicie su producción lingüística 
en el momento en el que se sienta listo o lista para hacerlo; esto, después de haber tenido exposición a la lengua por parte de una persona instructora. Al ser este un método en el que el estudiantado descubre y pone en práctica sus conocimientos a su propio ritmo, no se espera que exista un ambiente colaborativo en una clase en la que se trabaje con él.

El método de respuesta física total fue desarrollado por Asher en 1977 y según lo explica Alcalde (2011), "con este método se trabajan las acciones físicas, acompañadas de órdenes, bien por parte del profesor hacia los alumnos, bien al revés" (p. 17-18). En este método, según Brown (2001), se hace uso de comandos y se recomienda que sea utilizado con principiantes en el aprendizaje de una lengua, ya que pierde sus distintivos a medida que el estudiantado avanza en el aprendizaje de la lengua meta: "Enseñando por medio de comandos imperativos y de movimientos corporales, además de divertir, se puede dirigir la conducta del discente sin la necesidad de memorizar ni entender palabras en su totalidad" (Alcalde, 2011, p. 18). $\mathrm{Al}$ hacer uso de la respuesta física total, se promueve un aprendizaje dinámico en el cual el estudiantado va a reaccionar a estímulos orales que su docente se encargará de brindar, creando así un ambiente libre de presión que busca un aprendizaje más lúdico. Para este método, el estudiantado reacciona ante diversos estímulos, no necesariamente se promueve la colaboración entre los miembros de la clase; sin embargo, si se hace uso de ciertos juegos o actividades en equipo, podría existir colaboración y ayuda entre estudiantes.

Finalmente, el método comunicativo, desarrollado por Richards, según Hernández (2000), "en realidad no es un método sino un enfoque que entiende el aprendizaje de lenguas como un proceso donde lo más sustancial no son las formas lingüísticas, sino las intenciones comunicativas" (p. 148). Alcalde (2011) manifiesta que debido a los desaciertos de los otros métodos, se requería uno que "cubriese, mediante actividad, textos, diálogos y ejercicios variados y motivadores, todas las áreas en que se realiza la comunicación” (p. 16). Este método, continúa la autora, "logra su objetivo aplicando principios psicológicos y pedagógicos en el desarrollo de los materiales, consiguiendo alcanzar, a su vez, una armonía de componentes necesarios para el proceso comunicativo" (Alcalde, 2011, p. 16). Este enfoque, por tanto, busca el aprendizaje de la lengua extrajera de una forma significativa en la cual el estudiantado va a encontrar la utilidad del idioma en contextos reales.

El enfoque comunicativo es el que el Ministerio de Educación Pública de Costa Rica (MEP) indica que debe ser utilizado en este país; de hecho, el MEP señala en el Programa de estudio de inglés de III ciclo y educación diversificada (2001) que este enfoque crea un ambiente libre de estrés y brinda oportunidades para la interacción, hace uso de una metodología participativa y dinámica. Además de ello, el estudiantado debe hacer uso del pensamiento crítico para resolver problemas, trabajar en grupos, tomar riesgos, discutir diferentes temas, entre otras acciones. Por tanto, se puede apreciar que sí se busca crear un ambiente colaborativo de cierta forma.

Lo anterior muestra que a través de los años, quienes instruyen las lenguas han estado en el proceso de prueba y error en lo que a métodos o enfoques se refiere, cada uno de los métodos descritos, y los muchos otros que han existido, han gozado de gran popularidad en su momento; pero de la misma forma han perdido esta y han sido sustituidos por otros. Sin embargo, el profesorado de idiomas puede hacer uso de las técnicas y preceptos de cada uno 
de ellos durante su accionar pedagógico para lograr una mayor comprensión de los temas que le presentan a sus estudiantes.

Ninguno de los métodos anteriormente descritos ha probado ser perfecto y completamente eficaz; gran parte de ello se debe a que todas las personas somos diferentes y aprendemos de diversas maneras. Hernández (2000) ve el futuro de la enseñanza de las lenguas moviéndose hacia la idea de que el método ideal ya no existe, esta quedó muy atrás, llena de utopías; el futuro, según el autor, ve al educador y educadora vinculando la instrucción formal con la instrucción informal para así lograr un balance y un verdadero aprendizaje útil de la lengua.

El aprendizaje de una lengua extranjera lo que busca es que el estudiantado alcance un conocimiento de esta para así poder tener acceso a información especializada en esta lengua y ampliar sus horizontes profesionales y personales. Según apunta el gobierno de la ciudad autónoma de Buenos Aires en su diseño curricular de lenguas extranjeras para el año 2001, la enseñanza de lenguas extranjeras constituye un espacio que busca fomentar una actitud ética en lo que respecta a procesos de democratización social y cultural en la comunidad lingüística, saber que la otra persona existe. Esto va a promover la tolerancia y aceptación. De acuerdo con esta misma publicación, saber o conocer una lengua extranjera le permite al estudiantado poder escoger el contexto en cual puede usarla. Puede con esto hacer una utilización eficaz de la lengua en diversos contextos para diversos propósitos.

Dentro de las metodologías y estrategias didácticas existe un abanico interminable de opciones que en la actualidad puede utilizarse. Como una de las estrategias didácticas a utilizar se puede mencionar el aprendizaje colaborativo. Este, según Johnson (1993), a quien citan Collazos Guerrero y Vergara. (2002), es básicamente el uso de grupos de tal manera que el estudiantado, mediante el trabajo conjunto, logre maximizar su aprendizaje y el de los demás individuos. Esta, plantean Collazos et al (2002), es una estrategia que complementa el trabajo individual; la idea es básicamente lograr un conjunto que trabaja para la totalidad de miembros en busca de un fin común que engrandezca el proceso de aprendizaje.

Lo anterior viene a retar a los métodos de enseñanza que se han venido aplicando tradicionalmente, tales como el método de gramática-traducción, el método directo, entre otros ya mencionados que han asignado roles específicos tanto a docentes como a estudiantes y que, de cierta forma, han olvidado la importancia de la colaboración entre quienes aprenden una lengua.

La colaboración entre el estudiantado como parte de la metodología en los métodos o enfoques de enseñanza tradicionales, tal y como se describió anteriormente, no ha tenido una gran participación o protagonismo. De hecho, dentro de todos los métodos descritos únicamente aparece como una opción dentro del enfoque comunicativo. Este proyecto buscó, por lo tanto, experimentar algo diferente a lo que tradicionalmente se ha venido ofreciendo en materia de enseñanza de una lengua extranjera.

El aprendizaje colaborativo no viene a resolver los problemas que tanto el cuerpo docente como el estudiantado podría afrontar en un contexto académico sino, más bien, es una alternativa a considerar en el momento de enseñar un contenido o de poner este en práctica. Cabe destacar además que el estudiantado no aprende necesariamente por su condición de colectivo o que sea imposible aprender sin ayuda de otros u otras. Sin embargo, el aprendizaje cooperativo es una manera interesante y diferente que eventualmente puede traer muy buenos resultados, si se logran desarrollar actividades apropiadas y atinentes a este. 
La idea del aprendizaje colaborativo como estrategia de aprendizaje novedosa es la que mueve este proyecto. Se buscó trabajar con algo creativo, diferente y novedoso para motivar a sus participantes a mejorar las destrezas orales en la lengua inglesa. Esto responde principalmente al hecho de que en muchos de nuestros contextos educativos, muchas veces, en el ejercicio docente olvidamos la importancia del trabajo en equipo y el apoyo entre pares; prueba de ello es que tal y como se describió anteriormente, a través de varios métodos de enseñanza este aspecto ha sido obviado y se ha dado más énfasis al trabajo y al progreso individual.

Por tanto, el propósito de este artículo es describir la experiencia colaborativa vivida por estudiantes del curso LM-1230: Comunicación Oral I durante el primer semestre del año 2014. Este curso pertenece a la Escuela de Lenguas Modernas como parte del plan de estudios de la Carrera de enseñanza de inglés, que se imparte en la Sede del Pacífico de la Universidad de Costa Rica de manera desconcentrada. Este grupo de estudiantes trabajó actividades de manera colaborativa, poniendo en práctica la lengua que están aprendiendo (el inglés) para lograr cumplir con los objetivos de la clase.

\section{Situación de aprendizaje que se desea resolver}

En este aspecto es relevante proveer una descripción del estudiantado con el propósito de entender de una mejor forma la situación de aprendizaje a resolver. Este es un grupo de estudiantes compuesto por un total de 26 personas (20 mujeres y 6 hombres) con edades entre los 18 y los 24 años, todos de diferentes comunidades de Costa Rica tales como Malpaís, Orotina, Esparza, Monteverde, Palmares, San Ramón, Zarcero, entre otras. Todas estas localidades se caracterizan por pertenecer a regiones alejadas de la Gran Área Metropolitana del país, en su mayoría zonas con características rurales. El estudiantado de este curso manifiesta tener interés por el aprendizaje del inglés y la mayoría también posee mucho interés en enseñarlo, lo cual hace que se cuente con un grupo entusiasta y participativo.

Esta población ya ha pasado por un año completo en el que ha sido expuesta al idioma inglés en los cursos de Inglés Integrado I y II, en los cuales se le ha dado las herramientas necesarias para el adecuado desarrollo de sus habilidades comunicativas en el idioma inglés. De tal forma, estos y estas son estudiantes capaces de comunicar sus ideas en el idioma inglés. Basado en lo anterior, se puede decir que el estudiantado tiene la necesidad de incrementar su dominio oral de la lengua inglesa mediante la constante práctica tanto dentro como fuera de clases.

Los contenidos del curso giran en torno a dos grandes temas: salud y medio ambiente. La motivación en relación con estos contenidos se basa en la misma necesidad que se tiene de aprender y mejorar la expresión oral, la capacidad de generar opiniones y criterios propios que le hagan crecer y mejorar día tras día para poder culminar su proceso de aprendizaje y así desarrollarse de manera efectiva en el plano laboral, en este caso específico como docente de inglés.

Por tanto, la situación de aprendizaje por resolver es la imperante necesidad de buscar una técnica o estrategia distinta e innovadora que se salga de lo que la población ha venido trabajando en sus clases, que se han basado principalmente en clases magistrales por un lado y trabajo individualizado por otro. Con ello se pretende motivar al alumnado a aprender y a participar más en su proceso de enseñanza-aprendizaje. Se busca, además, que logren 
desarrollar aún más sus habilidades orales para poder alcanzar el nivel requerido para aprobar el curso y continuar su proceso de crecimiento académico dentro de su carrera.

\section{Descripción de la estrategia didáctica}

Esta es una estrategia didáctica que propone una serie de técnicas congruentes con el aprendizaje colaborativo. A continuación, se hace una descripción de la estrategia a utilizar así como cada una de las técnicas que se emplean y la fundamentación teórica que justifica no solo el uso del aprendizaje colaborativo, sino también la utilización de cada una de las actividades. Se establece el papel docente así como el estudiantil durante el proceso.

En la implementación de la estrategia didáctica se esperaba que los estudiantes y las estudiantes lograsen colaborar entre sí para alcanzar los objetivos de la actividad de la manera más apropiada que se pudiese, se tenía la expectativa de contar con un grupo de estudiantes participativo e interesado en el proceso y en el logro de metas concretas durante la clase. El docente, por su parte, sirvió como facilitador de la situación de aprendizaje y, a la vez, fue el encargado de manejar el tiempo y la secuencia de las actividades por realizarse dentro de la estrategia didáctica planteada.

Según lo establece Duca (2012), el trabajo docente es importante en diferentes momentos del proceso, ya que es quien debe plantear y diseñar las actividades a realizarse y buscar los recursos para su desarrollo. Además, es quien se debe encargar de motivar al estudiantado, ayudándolo y guiándolo en el proceso y, finalmente, es quien dará el seguimiento de la estrategia didáctica.

En lo que respecta al contexto en que se desarrollaron las actividades: se contó con un espacio amplio, con mobiliario apropiado y condiciones tanto de iluminación como de ventilación excelentes el lugar estuvo aislado de ruidos y problemas externos que pudieran, de una manera u otra, intervenir en el desarrollo de las actividades aquí propuestas.

El contenido seleccionado está dentro de los temas a desarrollar en el curso. Este curso posee dos ejes base: los contenidos de pronunciación y los temas de discusión. Los temas de discusión, por su parte, se dividen en dos grandes grupos: temas relacionados con la salud y temas relacionados con el medio ambiente. La estrategia se basó en los temas de medio ambiente. Propiamente, el tema tratado fue las especies animales en peligro de extinción, causas, consecuencias y acciones para evitarlo.

Como ya se mencionó, se buscó trabajar con el aprendizaje colaborativo por varias razones. Por ejemplo, el trabajo en equipo es vital para el desarrollo de habilidades y el logro de metas en la sociedad actual. Abrir la posibilidad de trabajar de esta manera durante la clase busca enseñar al estudiantado más que el solo contenido a desarrollar de tipo conceptual, sino también fomentar y desarrollar contenidos actitudinales en el logro de actitudes positivas hacia la colaboración, el trabajo en equipo, entre otros.

Según lo establece Scagnoli (2005), "el aprendizaje colaborativo es la instancia de aprendizaje que se concreta mediante la participación de dos o más individuos en la búsqueda de información, o en la exploración tendiente a lograr una mejor comprensión o entendimiento 
compartido de un concepto, problema o situación" (p. 1). En este caso, esta estrategia didáctica fue más que adecuada, ya que el contenido conceptual que se desarrolló fue el de las especies animales en peligro de extensión: causas, consecuencias y acciones para evitarlo. Estamos frente a un problema que tiene muchas aristas y en el cual existe una diversidad de criterios, unos hasta muy extremos.

El aprendizaje colaborativo buscó dar la oportunidad al estudiantado de poner en práctica sus habilidades orales en el idioma inglés mediante la discusión de un tema específico y le dio la oportunidad de expresar sus opiniones y puntos de vista alrededor de un tema que para algunas personas es controversial. Se buscó, además, el desarrollo de competencias lingüísticas en medio de una situación que le permitió al estudiantado cooperar o colaborar entre sí para alcanzar metas y objetivos comunes y poder crecer y ayudarse mutuamente.

Según lo apunta Scagnoli (2005), el aprendizaje colaborativo está inmerso en la teoría de constructivismo social y se basa en el proceso de construcción del conocimiento mediante el aprendizaje proveniente de la interacción con un grupo y mediante tareas realizadas en cooperación con otros. De acuerdo con Hsu (2002 citado por Scagnoli 2005), el objetivo de este tipo de aprendizaje es inducir al grupo de estudiantes a la construcción de su conocimiento a través de la exploración, la discusión, la negociación y el debate. Este tipo de actividades, además de crear una conciencia crítica alrededor de un tema, produce más oportunidades para el óptimo desarrollo de sus habilidades lingüísticas.

Cada una de estas técnicas formó parte de un conjunto de actividades conducentes al desarrollo de habilidades lingüísticas, haciendo un especial énfasis en la habilidad del habla. A su vez, todas forman parte de un aprendizaje colaborativo que no solamente busca el adecuado desarrollo de habilidades, sino que también se basa en la premisa de que somos seres sociales y como tales aprendemos mejor mediante la interacción y la colaboración.

De acuerdo con Collazos et al. (2002), el método de aprendizaje colaborativo se basa en la idea de que los estudiantes y las estudiantes deben trabajar en conjunto para aprender y, a su vez, son los responsables del aprendizaje propio y del de sus compañeros; se busca, entonces, un trabajo basado en el razonamiento, el autoaprendizaje y la colaboración.

Según lo establece Camarillo (2009), en la actualidad, en lo referente al campo laboral, cada día se necesita que quienes se profesionalizan posean más y mejores capacidades y habilidades entre las cuales, sin duda alguna, destaca la capacidad de organización, comunicación y trabajo en equipo. Por esta razón el aprendizaje colaborativo toma fuerza como una estrategia didáctica de gran utilidad.

Este tipo de aprendizaje se basa en dos premisas: la búsqueda de un consenso grupal en el cual se cuente con una participación de todos y todas, y lograr el compromiso y la realización de cada una de las actividades individuales que vayan a cumplir una función de carácter grupal.

Entre las ventajas que se pueden encontrar en el aprendizaje colaborativo está el esfuerzo conjunto, las relaciones positivas en el grupo de estudiantes y la salud mental que se garantiza mediante su interacción positiva.

Todas las técnicas que se propusieron (rompecabezas, creación de un collage, la lluvia de ideas, el debate y el mapa conceptual) buscaron el desarrollo de un efectivo trabajo grupal con 
la premisa de la colaboración. Cada una de estas técnicas tuvo una razón de ser dentro de esta estrategia didáctica. Por ejemplo, en el caso del rompecabezas, según lo establecen Aronson y Patnoe (1997), por décadas se ha utilizado la técnica del rompecabezas dentro del aprendizaje colaborativo, en esta experiencia, cada participante representa una pieza fundamental para la terminación y la comprensión de los logros finales del proceso. Cada una de estas piezas es esencial para lograr el objetivo final y esa es la manera en las que cada estudiante debe verse a sí: como parte de esta estrategia.

El uso del rompecabezas tradicional como actividad inicial de la clase buscó ejemplificar el proceso en el cual se iba a trabajar: cada estudiante es una pieza fundamental para el logro de los objetivos de la estrategia didáctica, por ende, no hubo una mejor manera de dar inicio a la clase que con una metáfora tan funcional. La estrategia del rompecabezas puso en evidencia lo que cada persona en una clase representa: una pieza útil y necesaria para completar acciones conducentes al aprendizaje grupal.

Por otra parte, hacer uso de un collage como técnica didáctica, y sin menospreciar su valor artístico, fue también parte de lo que se buscaba innovar en esta experiencia didáctica. Gallardo y Bellido (2003) consideran que el estudiantado encuentra en el collage un "medio de expresión cercano a la realidad que vive a diario, ya que le ayuda a expresarse manipulando papeles ricos en texturas, colores, formas, cuya ordenación responde a criterios tanto artísticos, como creativos" (p. 15). Los autores establecen que el collage se debe considerar "un medio de expresión creativo y didáctico" (Gallardo y Bellido, 2003, p. 15); este es además un proceso rico para trabajarse de manera colaborativa, ya que supone una interacción entre estudiantes que permite irle dando forma al pensamiento y a la creatividad. Gallardo y Bellido (2003) consideran que el collage permite la realización de trabajos colectivos mediante los cuales se puede observar la interacción entre el alumnado, evitando la competencia y fomentando la autorreflexión, el respeto, la participación, la colaboración y la comunicación.

Esta técnica es, por tanto, útil para el desarrollo del trabajo dentro del aprendizaje colaborativo. De hecho, se usó pensando no solo en las ventajas que como tal ofrece para el trabajo en equipo, sino que además promueve la creatividad y estimula los sentidos, con lo que se consigue un ambiente de trabajo agradable en el cual el grupo participante pudo colaborar basándose en sus propias habilidades. Resulta, entonces, una pieza clave en el desarrollo del trabajo que buscaba poner en práctica las habilidades lingüísticas del estudiantado, al interactuar durante el proceso creativo haciendo uso exclusivo de la lengua meta (el inglés).

En lo que respecta a la lluvia de ideas, según la Secretaria de Salud del Gobierno del Estado de Veracruz (2011), en su Plan de emergencia radiológica externo (PERE), esta es una técnica que se usa con frecuencia en las clases, debido a que propicia la generación de ideas que a su vez van a colaborar con un determinado propósito que es, básicamente, el proponer ideas o conocimiento de cada participante en relación con un tema o problema, para que en colectivo se logre una conclusión o un acuerdo común. Vista desde la perspectiva del aprendizaje colaborativo, esta técnica tiene gran utilidad debido a que busca una sana interacción de participantes para lograr un proceso colaborativo de generación de ideas en pro de lograr un objetivo común como grupo de trabajo.

La generación de ideas en pro de lograr un objetivo común es, precisamente, lo que se pensó al colocar esta actividad dentro de la estrategia didáctica: dar pie a la generación de ideas clases 
alrededor de un tema específico para luego abordarlo desde varios puntos de vista que fuesen socializados entre quienes participaron de la actividad, mediante un proceso de debates, el cual buscaba un compartir de opiniones y criterios provenientes de variedad de participantes para así poder contrastar criterios, siempre respetando y tolerando la diversidad de opiniones y criterios.

Según la Secretaría de Salud Gobierno del Estado de Veracruz (2011), el debate es ampliamente utilizado y consiste en un intercambio de ideas e información de un tema específico, este es un diálogo, una presentación y defensa de ideas y criterios, lo cual va a llevar a un pensamiento crítico. Esta técnica desarrolla habilidades lingüísticas y sociales, precisamente lo que se buscó en esta estrategia didáctica.

Teniendo como partida la lluvia de ideas para la generación de un diálogo en el cual se esperaba que quienes participaran del proceso hicieran uso de la lengua meta, se dio pie a un debate en el cual cada participante tenía la potestad de emitir sus criterios propios alrededor del tema a tratar. El debate permitió la participación espontánea del estudiantado, quienes defendían sus ideas y recibían además apoyo de sus pares, logrando con esto colaboración en su aprendizaje y un uso significativo de la lengua meta.

Finalmente, el mapa conceptual como técnica de enseñanza tiene mucho que aportar al proceso educativo. Esta es una actividad que permite la sistematización de la información y la representación gráfica de esta, de manera que lleve a una mayor comprensión de la información presentada. Según lo establecen Cañas y Novak (2013), los mapas conceptuales, cuando se elaboran a conciencia, revelan la organización cognitiva de los estudiantes y las estudiantes. A mayor eficiencia de parte del estudiantado en la construcción de sus mapas conceptuales, más se revela su nivel de comprensión y el nivel de sus errores.

De acuerdo con Díaz (2002), los mapas conceptuales -tal y como los desarrolla Novak en el año 1988- buscan facilitar al estudiantado las estrategias y condiciones que le lleven a aprender a aprender. Esto fue lo que se buscó conseguir al incluir esta técnica dentro de la estrategia didáctica: un aprendizaje significativo que reflejara capacidad de síntesis y comprensión de un tema específico, en el cual se hiciera uso exclusivo de la lengua meta. Un aprendizaje en el que además existiera un nivel de colaboración de apoyo de grupo que lograra facilitar el proceso como tal.

Como se puede observar dentro de lo propuesto en esta estrategia didáctica, tanto el estudiantado como el personal docente tuvieron un papel preponderante en la aplicación y desarrollo de cada una de las actividades o técnicas implementadas. El primer grupo es el partícipe principal del proceso, debió lograr un adecuado trabajo colaborativo con el fin de alcanzar metas propias y comunes que le atañen como grupo; en tanto que el docente fue quien debió fiscalizar todo el proceso y guiarlo de tal forma que, de cierto modo, también fuese visto como parte del proceso colaborativo.

\section{3- Aplicación de la estrategia didáctica}

Para lograr aplicar la estrategia didáctica se buscó hacer uso de una serie de técnicas específicas que fueran a servir a los participantes y las participantes para el desarrollo de sus habilidades. Tal y como se puede apreciar en el resumen a continuación, las actividades se desarrollaron durante una sesión de trabajo el día 26 de mayo del año 2014, en un espacio de 
tiempo correspondiente a 150 minutos y el orden de las actividades mantuvo una cronología lógica para garantizar el adecuado desarrollo de cada una de ellas.

A pesar de que el grupo constaba de 26 estudiantes matriculados, el día que se presentó la estrategia didáctica únicamente se contó con la presencia de 21 personas. Para la primera actividad, el grupo se dividió en 4 subgrupos, a cada subgrupo se le hizo entrega un rompecabezas que debía armar; al culminar este proceso se descubría la figura de una especie animal en peligro de extinción. Durante el desarrollo de esta actividad se observó la colaboración mutua en el proceso de ordenamiento de las piezas del rompecabezas. Hubo grupos que terminaron antes que otros y, lejos de simplemente no hacer más que esperar, miembros de varios subgrupos decidieron apoyar a los subgrupos que no habían podido culminar con la tarea asignada. La actividad tuvo una duración promedio de cinco minutos y el objetivo fue iniciar el proceso de colaboración en el estudiantado en una actividad relativa al tema a tratar a manera de introducción, además de ello, era importante que quienes tomaron parte en esta actividad hicieran uso exclusivo de la lengua inglesa.

Posterior a esta actividad introductoria, se le hizo entrega, a cada uno de los subgrupos, de información acerca de la especie animal que ya habían armado en el rompecabezas. Posterior a ello se les entregó materiales tales como tijeras, goma, papel periódico, revistas, periódicos viejos, entre otros. Esto, con el fin de que cada subgrupo realizara un collage que representara, de manera gráfica y sintetizada, la información que se les suministró acerca de la especie en extinción que ya habían conseguido identificar en el rompecabezas. La idea era que, mediante el collage, se expresaran detalles sobre la relevancia de la especie animal, su hábitat, el peligro actual al cual se ve expuesta, entre otros.

Se buscó como objetivo fundamental el realizar trabajo colaborativo para sistematizar la información y para la elaboración de un recurso visual para presentar la información ordenada y este se logró a cabalidad. Durante esta actividad, el estudiantado colaboró entre sí de tal forma que unos se encargaban de cortar y buscar imágenes representativas, otros las organizaban en el collage. Una vez finalizada la representación gráfica, la totalidad de integrantes del grupo pasaron al frente a exponer su trabajo justificando cada uno de los aspectos incluidos, asimismo brindó información acerca de la especie en extinción que se estaba representando.

La actividad numero tres consistió en una lluvia de ideas de la cual debieron ser partícipes todas las personas de la clase. Para la realización de esta técnica, quienes participaron debieron pasan a la pizarra y con un marcador empezar a escribir sus ideas acerca de las causas, consecuencias y soluciones que se le pueden brindar al maltrato animal y la extinción de especies animales. Con esta actividad se buscó trabajar en conjunto para exponer ideas generales acerca de un tema específico y con ello promover la discusión. Los estudiantes y las estudiantes participaron activamente y expusieron sus criterios y opiniones.

Luego de la lluvia de ideas, continuaron trabajando en subgrupos, en esta ocasión, se le entregó, a cada subgrupo, información acerca de las causas, consecuencias y soluciones para la extinción de especies animales. Cada grupo discutió estos aspectos. Posterior a ello se realizó un debate en el cual quienes participaron en la actividad plantearon sus ideas y opiniones en relación con el tema discutido para buscarle soluciones. El estudiantado debió colaborar entre 
sí para corregir y ayudarse mutuamente en aspectos relacionados con manejo del idioma meta La reacción ante esta tarea fue muy positiva, se logró una muy buena integración e interacción entre quienes participaron. Mientras preparaban y discutían el material asignado, se mantenía una conversación en cada subgrupo: cada miembro presentaba su opinión y su punto de vista y, posteriormente, se hizo una socialización de todos los aspectos discutidos dentro de cada uno de los subgrupos y se llegó a conclusiones importantes mediante un proceso colaborativo.

Esta estrategia se consideró importante pues permitió una interacción real entre quienes participaron de ella, se creó un espacio en el cual se compartieron opiniones sin temor alguno y se hizo uso de la lengua inglesa. Además de ello, existió apoyo entre quienes participaron en lo que respecta a la corrección de errores de gramática y pronunciación, cuando se hacía uso de la lengua meta. Fue una técnica apropiada para el nivel del estudiantado y permitió entender un problema en conjunto, al generar con ello opiniones individuales y colectivas que, posteriormente, fueron expuestas al resto de la clase. Con ello se logró un espacio apropiado para el desarrollo de sus habilidades lingüísticas.

Para poder sistematizar todo lo presentado y manifestarlo de una manera gráfica frente a toda la clase, cada uno de los subgrupos trabajó en una parte de un mapa conceptual que resumía todos los aspectos ya discutidos durante el debate. En este caso, es interesante ver que aún, a pesar de que el mapa conceptual se hizo por separado, cada subgrupo desarrolló un punto, al momento de unir las partes en la pizarra, todo tenía sentido. Esto garantizó el cumplimiento de los objetivos propuestos en cada una de las actividades anteriormente aplicadas, ya que la integración de los miembros de la clase fue tal que permitió una comprensión global del contenido que se trabajó y cada subgrupo logró plasmar opiniones con las que todas las personas en la clase estuvieron de acuerdo. Dicho acuerdo se alcanzó mediante el desarrollo de la estrategia didáctica en general.

Para finalizar la clase, se hizo un momento de reflexión en el cual el estudiantado presentó sus opiniones con respecto a las actividades desarrolladas durante esa sesión y, posterior a ello, cada estudiante, de manera individual, completó un instrumento elaborado por el docente. Este instrumento buscaba conocer la opinión del estudiantado con respecto a cada una de las actividades, a la estrategia didáctica en general, su reacción y opiniones así como sus recomendaciones, con el propósito de su valoración de la estrategia didáctica.

\section{4- Análisis de los resultados del proceso desarrollado}

\section{A. Bondades de la estrategia didáctica}

Esta estrategia didáctica a través de las cinco técnicas que se utilizaron permitió crear un vínculo de trabajo muy interesante en el estudiantado. La manera en que se desenvolvieron y lograron desarrollar cada una de las actividades fue muy buena, cabe señalar que se logró una participación activa de la totalidad de estudiantes presentes en la clase, se evidenció en el entusiasmo y la buena disposición por participar en cada una de las actividades que tuvo el grupo en general. 
Efectivamente, siempre existió colaboración entre los diferentes miembros del grupo, muy en especial cuando trabajaron en subgrupos más pequeños, los cuales permitían una interacción aún más profunda. Se vio como la totalidad de participantes colaboraron en los grupos de trabajo para ir cumpliendo las metas propuestas. Cuando se trabajó en subgrupos, cada miembro de estos estaba a cargo de una tarea específica, que realizaba y, a su vez, cuando terminaba, colaboraba con quienes no habían terminado de realizar su trabajo.

Cada miembro fue capaz de exponer su punto de vista alrededor del tema a discutir. De hecho, las discusiones previas al debate general en cada uno de los subgrupos fueron muy interesantes dado que se desenvolvieron en la lengua meta y además de eso se corregían y ayudaban mutuamente. Por tanto, esta es una estrategia que promueve integración y participación. Logra que el estudiantado ponga en práctica sus habilidades orales en el idioma inglés y, con ello, pueda desarrollar diferentes aspectos lingüísticos que les ayudasen a mejorar su nivel en esta lengua.

\section{B. Limitaciones}

Esta estrategia, si bien promueve una amplia participación del estudiantado, tiene detalles que podrían mejorarse. Por ejemplo, al estar todo el grupo trabajando en conjunto, es casi inevitable el hecho de que en ciertos momentos va a existir ruido interno. Quienes participan hablan y colaboran entre sí, logran acuerdos, trabajan en tareas específicas y, por ende, se puede generar un poco de desorden; pero esto tampoco va a detrimento de la actividad o del cumplimiento de los objetivos.

En alguna ocasión, o en alguna de las tareas puede ser que alguno o alguna de los miembros de los subgrupos simplemente decidan no hacer nada y permitir que sean sus compañeros y compañeras quienes se encarguen del trabajo. Esta es una situación que puede ocurrir y para ello es importante informarles, desde el comienzo, de las actividades y acerca de la importancia que tiene para el éxito de la actividad la participación activa de cada miembro del grupo, logrando así que se motiven entre sí y que motiven a las otras personas a hacer su trabajo de manera eficiente. Esta situación de ruido, dichosamente, no ocurrió durante la aplicación de la estrategia didáctica; sin embargo, debido a sus características es algo que eventualmente podría suceder.

\section{Proyecciones del uso}

El uso del aprendizaje colaborativo en la rama de la enseñanza y el aprendizaje de una lengua, en especial el inglés, tiene una amplia utilidad. Para aprender una lengua no hay mejor recomendación que practicarla y para lograr una práctica efectiva se necesita de la interacción entre individuos.

El uso de técnicas que respondan y promuevan el aprendizaje colaborativo genera interacción y trabajo en equipo entre el estudiantado, lo cual garantiza la puesta en práctica de las estructuras lingüísticas. Al realizar la práctica oral en grupo se genera una mejora en las estructuras gramaticales y la pronunciación de palabras y sonidos, ya que se promueve la corrección y la ayuda por parte de quienes participan en el trabajo; además de ello, se logra 
mejorar el vocabulario, pues el banco de palabras del que hace uso una persona es de cierta forma muy propio y limitado y, si no existe interacción con otros individuos, es muy difícil hacerlo crecer y variar.

Por otra parte, considero que esta estrategia didáctica tiene la ventaja de que puede ser utilizada casi en cualquier contexto y en cualquier nivel. Cualquier tema se puede desarrollar mediante las técnicas que se usaron en esta estrategia, únicamente se deben hacer las modificaciones en cada caso. De igual forma, las técnicas ya descritas pueden adaptarse a las necesidades de cada nivel, así entonces, se pueden trabajar con estudiantes principiantes, intermedios o avanzados. Esta característica, considero yo, hace que esta estrategia sea muy útil, ya que podemos hacerla responder a diferentes necesidades.

Finalmente, otra ventaja que desde mi perspectiva de docente le encuentro al aprendizaje colaborativo es el hecho de que minimiza la figura del personal docente en la clase y le da mucho más valor a la figura estudiantil. Este es una guía y un moderador en el proceso; pero no tiene el papel protagónico, este lo asume el estudiantado a quien, además, se le encomienda la tarea de ayudarse mutuamente.

\section{Valoración de la estrategia desde la perspectiva docente}

Desde la perspectiva del docente, esta estrategia didáctica es efectiva. Todas las actividades realizadas gozaron de buena aceptación por parte del estudiantado y promueven la participación activa en la clase.

Algo que es incluso más interesante es el hecho de que una parte del grupo de estudiantes, que en situaciones normales siempre se han mantenido más renuentes a hablar y participar en clase, durante la aplicación de la estrategia didáctica lo hicieron y lo hicieron muy bien, sobre todo cuando trabajaron en los subgrupos. Parece que los grupos más pequeños abren la posibilidad de perder el temor y ayudan a que personas que generalmente son tímidas en clase mejoren su autoestima y se sientan capaces de participar eficientemente.

Esta estrategia es fructífera desde diferentes puntos de vista, no solo promueve participación activa y ayuda a estudiantes a tomar valor y hacer las cosas, si no que además promueve el trabajo en equipo, la colaboración y la camaradería. De esta forma, además de estar trabajando un tema como lo es los animales en peligro de extinción, se están desarrollando ejes trasversales en los cuales valores como la cooperación son enfatizados.

En efecto, el aprendizaje colaborativo, además de permitirnos desarrollar cualquier contenido, tiene la ventaja de que de manera indirecta promueve una serie de valores que van a producir una mejora en las relaciones del grupo de estudiantes, la confianza de los miembros que lo integran y su capacidad de trabajo.

\section{E. Valoración de la estrategia desde la perspectiva estudiantil}

Según el instrumento antes mencionado en la descripción de la estrategia didáctica y que se aplicó a la totalidad de participantes de la clase, hay muchos aspectos que se pueden discutir, por ejemplo, de 21 participantes, 18 consideraron que la sesión en la cual se trabajó el aprendizaje colaborativo fue muy interesante y solamente 3 consideran que fue interesante. 
La actividad que más gustó fue la creación del collage. Esto, según manifiestan, se debe al hecho de que todos y todas colaboraron en la creación, les sirvió para sintetizar ideas, promueve interacción, se puede apreciar diversos puntos de vista, cada quien fue libre de aportar ideas y pensamientos, se pueden expresar mejor las ideas, entre otras. Seguido del collage, se menciona el uso del mapa conceptual, el debate y la lluvia de ideas, el rompecabezas es la actividad que cuenta con menos apoyo. Entre las razones que favorecen a las otras actividades están el hecho de que promueven colaboración entre los miembros del grupo, ayudan a expresar ideas y a sintetizarlas.

Cuando se consulta acerca de la efectividad del aprendizaje colaborativo en el proceso de enseñanza-aprendizaje, la totalidad de participantes considera que sí funciona, ya que promueve el compartir de conocimientos, ideas, experiencias, ayuda a entender más a los demás y, por ende, promueve valores como el respeto y la tolerancia, se aprende de las demás personas y permite perder el miedo a participar.

$\mathrm{Al}$ consultarles al grupo de participantes acerca del aspecto o los aspectos que modificarían de la sesión de aprendizaje colaborativo de la cual participaron, la totalidad responde que no cambiaría nada, ya que consideran que todas las actividades fueron efectivas para su aprendizaje. Además plantean que es importante promover el uso de actividades colaborativas en clases futuras.

\section{F. Consideraciones finales del docente}

Desde mi perspectiva personal, como docente y promotor de la actividad, considero que la estrategia es efectiva, que el aprendizaje colaborativo es útil y perfectamente aplicable a casi todo contexto educativo. Los seres humanos no somos entidades aisladas, dependemos del contacto, de la colaboración de otros individuos para poder potenciarnos más. Es ilógico guardar todo nuestro conocimiento para nosotros en lugar de compartirlo y colaborar con las demás personas.

La colaboración y el trabajo en equipo promueven más que conocimiento, favorecen valores que eventualmente van a ser muy útiles para el estudiantado en un futuro profesional, pues en la labor docente es casi imposible pretender que se va a accionar de manera aislada, por ende, aspectos como el trabajo en equipo y la colaboración deben ser fomentados durante la formación profesional de futuros y futuras docentes.

Además de todo lo ya dicho, es importante promover diferentes actividades en clases; se necesita, además de explorar el trabajo en equipo, promover la creación artística, ya que actividades de este tipo brindan la posibilidad de expresarse de una forma diferente y muy útil.

La historia de la enseñanza, particularmente en este caso la enseñanza de las lenguas, nos ha mostrado que quienes se han encargado de esta han estado en una constante búsqueda de métodos, estrategias e ideas que hagan de su trabajo algo más sustancial, atractivo y significativo para sus estudiantes: he ahí la enorme cantidad de métodos y enfoques que se han desarrollado. Mi opinión al respecto es simple: no existe un método infalible, de hecho no todos los métodos se pueden aplicar a todos los contextos y a todas las poblaciones estudiantiles; pues todos son distintos. Esto nos reta, como educadores y educadoras, a investigar, leer, innovar, compartir: a darle un valor agregado a nuestro trabajo en busca del desarrollo y cumplimiento de objetivos de la población con la que trabajemos. 
Todos los métodos tienen algo bueno y provechoso, la clave está en descubrir cuál parte puede servir para la población de estudiantes a cargo. La única manera de lograr esto es conocer al estudiantado y probar. Démosle algo más a nuestra labor docente, sorprendamos a nuestro estudiantado día tras día y dejémonos sorprender por este.

\section{Conclusiones}

Como personal docente, estamos llamados, según lo que establece Duca (2012), a planear y diseñar actividades para motivar al estudiantado, ayudarle y guiarlo en el proceso. Esto es precisamente lo que esta estrategia didáctica buscó: proponer algo diferente por la parte docente, para guiar al estudiantado a nuevas experiencias de aprendizaje: que además de enseñarle pudiesen motivarle a seguir aprendiendo.

El aprendizaje colaborativo, al ser una instancia de aprendizaje concretada "mediante la participación de dos o más individuos en la búsqueda de información” (Scagnoli, 2005, p. 1), logra, mediante la implementación de actividades colaborativas dentro de la clase de inglés, la meta propureta. Se tuvo a la totalidad de la población trabajando y colaborando entre sí para alcanzar un objetivo común. Se logró colaboración, trabajo en equipo y una participación efectiva de la totalidad de la clase.

Scagnoli (2005) destaca el hecho de que el aprendizaje colaborativo busca construir conocimiento a partir de la interacción de grupo y de la cooperación. Toda la clase se enfocó hacia la discusión de un problema y con cada uno de los pasos y las actividades realizadas se generaron conclusiones; cada miembro del grupo logró aportar su propio criterio alrededor del tema; por tanto, se logró construir conocimiento valioso y con esta construcción de conocimiento el estudiantado pudo poner en práctica sus habilidades lingüísticas en el idioma inglés.

Collazos et al. (2002) destacan el hecho de que el aprendizaje colaborativo se basa en la idea de que el estudiantado debe trabajar junto para así aprender, además de ello, son responsables de su aprendizaje y del de sus compañeros y compañeras. Ante este enunciado es interesante destacar el hecho de que en efecto, mediante la aplicación de las actividades colaborativas, se observó que hubo mucha cooperación en el grupo, cuando alguien tenía que recibir ayuda, sus compañeras y compañeras estaban ahí. Se observó, además, como entre participantes se corregían sus errores mutuamente.

En el caso que nos ocupa, la presencia del docente funcionó, meramente, como una guía o mediación: en realidad el aprendizaje colaborativo minimiza el rol del docente y maximiza el trabajo del estudiantado. A través de las actividades realizadas se observó independencia y trabajo en equipo. Todas las actividades implementadas fueron útiles y eso fue fácilmente apreciable.

El aprendizaje colaborativo, basado en lo observado durante la implementación de las actividades descritas en este documento, es útil; genera muchos beneficios positivos entre quienes participan de él y puede ser fácilmente adaptado a muchos contextos educativos. Además de ello, se sale de los moldes educativos que muchas veces ha recetado la academia. Es algo más, hace la diferencia en el proceso de enseñanza-aprendizaje y el estudiantado lo siente y lo disfruta. 


\section{Referencias}

Alcalde, N. (2011). Principales métodos de enseñanza de lenguas extranjeras en Alemania. Revista de Lingüistica y Lenguas Aplicadas, 6, 9-23. doi: http://dx.doi.org/10.4995/rlyla.2011.878

Álvarez, M. (2013) Análisis de las metodologías para la enseñanza de la lengua inglesa en educación secundaria (Tesis de maestría). Universidad de Almería, España). Recuperado de http://repositorio.ual.es:8080/jspui/bitstream/10835/2391/1/Trabajo.pdf

Aronson, E., y Patnoe, S. (1997). The jigsaw classroom: Building cooperation in the classroom [La clase del rompecabezas: Construyendo cooperación en la clase] (2 ${ }^{\text {da }}$ ed.). New York: Addison Wesley Longman.

Brown, D. (2001). Teaching by Principles an Interactive Approach to Language Pedagogy [Enseñando por principios un enfoque interactivo para la pedagogía de la lengua] (2da ed.). White Plains, N.Y: Longman.

Camarillo, J. (2009). Promoviendo el aprendizaje colaborativo en la materia de diseño de conceptos de moda infantil, mediante el uso de modelos o maniquíes infantiles. En Universidad Autónoma de Aguascalientes (Ed.), Proyectos de innovación educativa: Una oportunidad para realizar las propuestas de los profesores (pp.15-46). Recuperado de www.uaa.mx/direcciones/dgdp/defaa/descargas/proys 09-10.pdf

Cañas, A. y Novak, J. (2013). Estrategias para iniciar a los estudiantes de primaria/ secundaria en la elaboración de mapas conceptuales. Eduteka. Recuperado de http:// www.eduteka.org/MapasConceptuales.php

Collazos, C., Guerrero, L. y Vergara, A. (2002) Aprendizaje colaborativo: Un cambio en el rol del profesor. Recuperado de http://users.dcc.uchile.cl/ luguerre/papers/CESC-01.pdf

Díaz, J. (2011). Los mapas conceptuales como estrategia de enseñanza y aprendizaje de la educación básica. Educere, la revista Venezolana de educación, 6(18), 194-203. Recuperado de http://www.redalyc.org/pdf/356/35601811.pdf

Duca, M. (2012). El aprendizaje colaborativo (Tesis de licenciatura). Universidad Fraternidad de Agrupaciones Santo Tomás de Aquino, Argentina. Recuperado de http://www. biblioises.com.ar/Contenido/300/370/Aprendizaje\%20colaborativo.pdf

Gallardo, P. y Bellido, M. (2003). Aspectos didácticos del collage y del cartel publicitario a través de la educación multicultural. Recuperado de http://www.juntadeandalucia.es/ educacion/portalaverroes/documents/10306/1585186/XVI ADO Aspectos+did\%C3\%A1 $\underline{\text { cticos }+ \text { del+collage }+y+d e l+c a r t e l+\text { publicitario.pdf }}$ 
Gobierno de la Cuidad Autónoma de Buenos Aires, Secretaría de Educación, Dirección de Currícula. (2001). Diseño curricular de lenguas extranjeras niveles 1, 2, 3 y 4. Buenos Aires, Argentina. Recuperado de www.buenosaires.gob.ar/areas/educacion/curricula/dle web.pdf

Hernández, F. (2000). Los métodos de enseñanza de lenguas y las teorías de aprendizaje. Revista de investigación e innovación en la clase de idiomas, 11, 141-153. Recuperado de http://encuentrojournal.org/textos/11.15.pdf

Jiménez, A. (2010). La expansión colonial y el reparto del mundo. Revista innovación y experiencias educativas, 30. 1-11. Recuperado de http://www.csi-csif.es/andalucia/ modules/mod ense/revista/pdf/Numero 30/ANDRES MANUEL JIMENEZ BALLESTEROS 01.pdf

Marín, E. (2012). Cronología de la enseñanza del inglés en Costa Rica durante el siglo XX. Revista Comunicación, 33(21), 4-15. Recuperado de http://revistas.tec.ac.cr/index.php/ comunicacion/article/viewFile/809/723

Martín, M. (2009). Historia de la metodología de la enseñanza de lenguas extranjeras. Revista Tejuelo, 5, 54-70. Recuperado de dialnet.unirioja.es/descarga/articulo/2983568.pdf

Ministerio de Educación Pública. (2001). Programas de Estudio de Inglés III ciclo y educación diversificada. San José: MEP

Muñóz, A. (2010). Metodologías para la enseñanza de las lenguas extrajeras: Hacia una perspectiva crítica. Revista Universidad EAFIT, 46(159), 71-85. Recuperado de http:// publicaciones.eafit.edu.co/index.php/revista-universidad-eafit/article/viewFile/1065/959

Secretaria de Salud Gobierno del Estado de Veracruz. (2011). Plan de Emergencia Radiológica Externo (PERE): Curso de Actualización para Instructores del PERE 2011, Catálogo de técnicas didácticas [en línea]. Veracruz, México. Recuperado de https://www.academia. edu/4976402/Curso de Actualizaci\%C3\%B3n para Instructores del PERE 2011

Quezada, R. (2011). Biografía de la lengua Inglesa: Cómo nació, a qué se dedica, y... ¿Pensando en retirarse? Revista Destiempos, 32, 3-17. Recuperado de http://www.destiempos.com/ n32/rquezada.pdf

Scagnoli, N (2005). Estrategias para motivar el aprendizaje colaborativo en cursos a distancia. Recuperado de https://www.ideals.illinois.edu/bitstream/handle/2142/10681/ aprendizaje-colaborativo-scagnoli.pdf?sequence $=4$

Silva, M. (2006). la enseñanza del inglés como lengua extranjera en la titulación de filología inglesa: El uso de canciones de música popular no sexista como recurso didáctico (Tesis doctoral). Universidad de Málaga, España. Recuperado de http://www.biblioteca.uma.es/ bbldoc/tesisuma/16853805.pdf 